\title{
Haben die Russen Studien getürkt?
}

\author{
Der wissenschaftliche Ost-West-Konflikt um eine russisch-amerikanische Herzinsuffizienz-Studie ist \\ aufgelöst: Nach dreijährigen Nachforschungen ist die Ursache der diskrepanten Ergebnisse geklärt.
}

_ In der randomisierten, doppelblinden TOPCAT-Studie [Pitt et al. N Engl J Med. 2014;370:1383-92] erhielten herzinsuffiziente Patienten mit noch normaler linksventrikulärer Auswurffraktion neben der üblichen Medikation entweder 15-45 mg Spironolakton $(\mathrm{n}=1.722)$ oder Placebo $(\mathrm{n}=1.723)$. 1.678 Patienten kamen aus Russland und Georgien, 1.767 aus den USA, Kanada, Brasilien und Argentinien. Nach 3,3 Jahren gab es keinen Unterschied beim Endpunkt aus kardiovaskulär bedingtem Tod, überlebtem Herzstillstand und Klinikaufnahme wegen Herzinsuffizienz.

Eine spätere Analyse [Pfeffer et al. Circulation. 2015;131:34-42] zeigte, dass die amerikanischen Probanden signifikant von Spironolakton profitierten, nicht aber die aus den östlichen Ländern.
Man vermutete West-Ost-Unterschiede beim Schweregrad der Herzinsuffizienz, bei den demografischen Daten und/oder der Wirkung von Spironolakton.

Nun zeigt eine neue Analyse eine bemerkenswerte, wenn auch banale Erklärung. Blutproben von 76 Patienten aus den USA und Kanada sowie 66 Patienten aus Russland wurden auf Canrenon, den aktiven Metaboliten von Spironolakton, untersucht. Alle Patienten waren der Verumgruppe zugeteilt und hatten die regelmäßige Einnahme des Medikaments bestätigt. In der Analyse war der Metabolit allerdings bei 30\% der Russen nicht nachweisbar. Bei den Amerikanern waren es hingegen nur 3\%.

- de Denus S, O'Meara E, Desai AS et al. Spironolactone metabolites in TOPCAT: new insights into regional variation. New Engl J Med. 2017:376:1690-2

\begin{abstract}
KOMMENTAR
Für große Studien werden immer häufiger Patienten aus vielen Ländern, teils weltweit rekrutiert. Das verkürzt die Dauer der Rekrutierungsphase und der Studie insgesamt. Auch Teile der Arzneimittelprüfung werden regelmäßig ins Ausland verlegt, z. B. nach Indien. Allerdings gibt es teils gravierende regionale Unterschiede hinsichtlich der ärztlichen Routine, der Definition von Krankheitsbildern wie der Herzinsuffizienz, des Gesundheitssystems und der Patienteneigenschaften. Die hoffnungsvolle Erwartung, dass die Ergebnisse auf die meisten Patienten weltweit übertragbar sind, wird durch die jahrelangen Posthoc-Analysen der TOPCAT-Studie eindrücklich konterkariert.
\end{abstract}

Prof. Dr. med. H. Holzgreve

\section{So beeinflusst Coca-Cola den Wissenschaftsjournalismus}

Die seit Jahren durch die industrialisierte Welt schwappende Adipositaswelle bedroht die Umsätze der Hersteller von zuckerhaltigen Getränken. So wird in Großbritannien ab 2018 die auch in Deutschland heiß diskutiert Zuckersteuer eingeführt. Die Werbung von CocaCola, dem weltgrößten Hersteller von Zuckerlimo, zielt schon seit einigen Jahren darauf ab, dass Übergewicht kein Problem der Nahrungszufuhr, sondern der mangelnden sportlichen Aktivität sei. Wer ständig Sport treibt, steckt die Kalorien nicht nur locker weg, er braucht sie sogar zur Erfrischung. So greifen denn die sehnigen schweißnassen Hobbyathleten in den Spots zur kühlen Flasche mit den wunderbaren Kondenswasserperlen (Abb.).

Ganz ähnlich wie früher die Tabakindustrie spannt Coca-Cola auch die Wissenschaft und den Wissenschaftsjournalismus in diese Kampagne ein. So spendete die Firma 1 Million US-Dollar an die Universität von Colorado - die Wirkungsstätte von James Hill, Präsident des "Netzwerks für die globale Energiebilanz" und be-

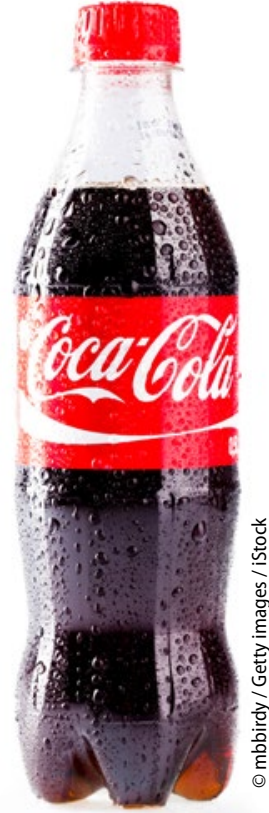

Ein Sportgetränk? zeichnenderweise ein Pädiater, der den Interessen der Firma den vermeintlich wissenschaftlichen Adel verlieh. Nach einem kritischen Artikel in der "New York Times" hat die Universität diese Summe aber wieder zurückgezahlt.

Über dunkle Kanäle wurde nun auch bekannt, dass Journalisten zu mehreren Konferenzen der Universität eingeladen wurden, auf denen sie von den „Rockstars der Adipositasforschung“ über die wahren Ursachen der gewichtigen Welle „aufgeklärt" wurden. Unter anderem führte dies zu einem TV-Beitrag auf CNN, der Übergewicht auf Bewegungsmangel, nicht auf Zuckergetränke zurückführte.

Wieder einmal sehen wir hier: „Cui bono?" und „Wer finanziert?" sind wichtige Fragen, um die Hintergründe der uns präsentierten Informationen einschätzen zu können.

Prof. Dr. med. H. S. FüeßI

- Thacker P. Coca-Cola's secret influence on medical and science journalists. BMJ. 2017;357:j1638 